\title{
Contributions to single-shot energy exchanges in open quantum systems
}

\author{
R. Sampaio,,${ }^{1, *}$ J. Anders, ${ }^{2}$ T. G. Philbin, ${ }^{2}$ and T. Ala-Nissila ${ }^{1,3}$ \\ ${ }^{1}$ QTF Center of Excellence, Department of Applied Physics, Aalto University, P. O. Box 11000, FI-00076 Aalto, Finland \\ ${ }^{2}$ CEMPS, Physics and Astronomy, University of Exeter, Exeter, EX4 4QL, United Kingdom \\ ${ }^{3}$ Interdisciplinary Centre for Mathematical Modelling and Department of Mathematical Sciences, Loughborough University, Loughborough, \\ Leicestershire LE11 3TU, United Kingdom
}

(Received 14 September 2018; published 25 June 2019)

\begin{abstract}
The exchange of energy between a classical open system and its environment can be analyzed for a single run of an experiment using the phase-space trajectory of the system. By contrast, in the quantum regime such energy exchange processes must be defined for an ensemble of runs of the same experiment based on the reduced system density matrix. Single-shot approaches based on stochastic wave functions have been proposed for quantum systems that are continuously monitored or weakly coupled to a heat bath. However, for systems strongly coupled to the environment and not continuously monitored, a single-shot analysis has not been attempted because no system wave function exists for such systems within the standard formulation of quantum theory. Using the notion of the conditional wave function of a quantum system, we derive here an exact formula for the rate of total energy change in an open quantum system, valid for arbitrary coupling between the system and the environment. In particular, this allows us to identify three distinct contributions to the total energy flow: an external contribution coming from the explicit time dependence of the Hamiltonian, an interaction contribution associated with the interaction part of the Hamiltonian, and an entanglement contribution, directly related to the presence of entanglement between the system and its environment. Given the close connection between weak values and the conditional wave function, the approach presented here provides a new avenue for experimental studies of energy fluctuations in open quantum systems.
\end{abstract}

DOI: 10.1103/PhysRevE.99.062131

\section{INTRODUCTION}

Open quantum systems are ubiquitous in realistic physical scenarios such as novel quantum devices and quantum computation, and a proper understanding of their behavior is of both conceptual and practical importance. The focus of open quantum systems is to describe the nonunitary dynamics of a system embedded in a larger environment. In principle this can be done through the system's reduced density operator, which contains the information required to compute the statistics of any observable of the system. However, in dealing with large environments such as an infinite heat bath, the exact evolution of the reduced density operator is not available and approximations must be employed [1]. For example, the popular Lindblad master equation for Markovian dynamics [2] can be derived under the assumption of weak coupling of the system to the environment and a clear separation of timescales $[1,3]$. Energy exchange between the system and its environment can then be studied at the level of the system's reduced density operator [4].

Recently, there has been growing interest in understanding the fluctuation of energy in open systems at the single-shot level of individual runs of an experimental protocol [4-12]. This scenario is commonly described by unraveling master equations, leading to a dynamical equation for the evolution

*rui.ferreirasampaio@ aalto.fi of a stochastic wave function $[1,13]$. This stochastic state is used as a numerical tool to calculate the density operator but it contains information beyond it. In particular, approaches based on the quantum-jump method [14] have facilitated the definition of stochastic thermodynamic quantities in an effort to extend the framework of classical stochastic thermodynamics to the quantum regime. The explicit time dependence of the Hamiltonian is typically understood as the external work while jumps are associated with some form of heat or entropy exchange due to the interaction with the environment [5-7,9,15-18].

In order to have a well defined wave function in the standard interpretation, quantum-jump approaches must be rooted in continuous measurement schemes [19,20]. For systems weakly coupled to large environments where Markovian master equations apply, this is not problematic since the reduced density matrix associated with the continuously monitored process is the same as that of the unmonitored process [19] (see, however, Refs. [21,22]). However, for small environments or strong interactions, continuous monitoring of the system or environment will induce an evolution that can significantly differ from that of the undisturbed process. In particular, this will destroy the correlations due to entanglement, preempting the study of its effects on energy fluctuations. Moreover, even for the continuously monitored process, strong interactions can lead to non-Markovian evolution for which quantum-trajectories approaches are not consistent with the standard interpretation of quantum mechanics $[21,22]$. 
In this paper our aim is to go beyond the weak-coupling approximation and continuously monitored systems to fully uncover the single-shot contributions to the energy exchange of a quantum system that arise due to its interaction and/or entanglement with its environment. As we point out above, tackling this question within the standard formulation of quantum mechanics is challenging because a system that is entangled with its environment cannot be described by a wave function of its own, i.e., there is no system wave function that depends only on the degrees of freedom of the system [20]. Within this formulation, the most detailed description of a system is in terms of its reduced density matrix which is in general a mixture of wave functions. A wave function for an open quantum system is here only admissible in the limit of a continuously measured environment and Markovian evolution of the system [21,22].

In contrast it has been shown that a unique conditional wave function (CWF) [23] can be identified for a quantum system that is entangled with its environment within the Bohmian configuration space formulation of quantum mechanics [23-25]. Conditional wave functions and other Bohmian related approaches [26-30] have become a tool for the investigation of transport in nanoscale electronic system [31-34], chemical reactions [35,36], tunneling [27], and the description of experiments such as the double slit [37-39], nonlocal steering [40], and spin measurements [41]. A review on different applications can be found in Ref. [42].

Here we employ the conditional wave function and its time evolution to define a conditional energy associated with a single-shot experiment and establish a formally exact analytic expression for energy exchanges during the joint systemenvironment time evolution. We show that the rate of energy exchange naturally partitions into three terms that can be interpreted as an external, an interaction, and an entanglement contribution. Each of these terms can be nonzero while the others vanish, e.g., if the system and environment are entangled but there is no interaction term between them, then the entanglement contribution is nonzero while the interaction contribution is zero. By explicitly solving a few simple model systems we illustrate the behavior of these terms for various scenarios of driven open systems. In contrast to many previous studies, the results presented here are valid for arbitrary environments-not just heat baths-and general Hamiltonians, including time-dependent interactions. In other words, no assumptions or approximations need to be made about the environment or the system's coupling to it, and also no coarse-graining (partial tracing) is needed. The results provide a direct link between entanglement and energy fluctuations at the level of individual runs of an experiment. Given the close relationship between CWFs and weak measurements [43] this provides a new avenue for empirical inquiry into the role of entanglement in energy fluctuations in open systems.

The paper is organized as follows. In Sec. II we review the definition of the conditional wave function and its dynamics under a generalized Schrödinger equation. In Sec. III we formally define the conditional energy and show how its time derivative leads to three different contributions to the energy exchange, with different physical interpretations. In Sec. IV we analytically solve a number of examples to illustrate how these different contributions manifest themselves in concrete settings. We provide a generalization to mixed states in the Appendix and summarize our findings in Sec. V.

\section{CONDITIONAL WAVE FUNCTIONS}

Here we give the definition and some intuition for the concept of CWF. A detailed discussion on the CWF and its derivation can be found in, e.g., Ref. [23]. We will then derive the nonlinear Schrödinger equation (NSE) that dictates its evolution, a key ingredient in identifying the different contributions to the energy fluctuations.

\section{A. Definition}

We consider a system of $N$ nonrelativistic point particles in the Bohmian approach with a general Hamiltonian $\hat{H}(t)=$ $\sum_{i=0}^{N-1} \hat{P}^{(i)^{2}} /\left[2 m^{(i)}\right]+V\left[\left\{\hat{Z}^{(i)}\right\}, t\right]$, where $\hat{P}^{(i)}$ and $\hat{Z}^{(i)}$ are the three-dimensional momentum and position operators of particle $i$, respectively; $m^{(i)}$ is the mass of particle $i$; and $V$ is a function of the operators $\hat{Z}^{(i)}$ and time only. In this case, the $j$ Cartesian component of the velocity field of particle $i$ is given by [44-46]

$$
\begin{aligned}
v_{j}^{(i)}(\vec{z}, t) & =\frac{1}{m^{(i)}} \operatorname{Re}\left\{\frac{\left\langle\vec{z}\left|\hat{P}_{j}^{(i)}\right| \Psi(t)\right\rangle}{\langle\vec{z} \mid \Psi(t)\rangle}\right\} \\
& =\frac{1}{2 m^{(i)}} \frac{\operatorname{Tr}\left[\hat{\Pi}(\vec{z})\left\{\hat{P}_{j}^{(i)}, \hat{\sigma}^{(\Psi)}(t)\right\}\right]}{\operatorname{Tr}\left[\hat{\Pi}(\vec{z}) \hat{\sigma}^{(\Psi)}(t)\right]},
\end{aligned}
$$

where $\hat{\sigma}^{(\Psi)}(t)=|\Psi(t)\rangle\langle\Psi(t)|$ with $|\Psi(t)\rangle$ the wave function of the combined system and environment, $\{\cdot, \cdot\}$ is the anticommutator, $\vec{z}:=\left(\vec{z}^{(0)}, \ldots, \vec{z}^{(N-1)}\right)$ is a point in the configuration space of the $N$ particles with $\vec{z}^{(i)}$ the three-dimensional position of particle $i$, and $\hat{P}_{j}^{(i)}$ is the momentum operator associated with the $j$ th component of the momentum of particle $i$ and $\hat{\Pi}(\vec{z}):=|\vec{z}\rangle\langle\vec{z}|$, where $|\vec{z}\rangle$ is the basis vector associated with the configuration point $\vec{z}$. Note that $v_{j}^{(i)}(\vec{z}, t)$ is proportional to the real part of the weak value of the momentum operator $\hat{P}_{j}^{(i)}$ with preselection on $|\Psi(t)\rangle$ and postselection on position $[46,47]$ (see below). Integrating the above equation yields a formal expression for the trajectory of each particle as

$$
\vec{Z}^{(i)}\left(t \mid \vec{z}_{0}\right)=\vec{Z}^{(i)}\left(0 \mid \vec{z}_{0}\right)+\int_{0}^{t} \vec{v}^{(i)}\left[\vec{Z}\left(s \mid \vec{z}_{0}\right), s\right] d s,
$$

where $Z^{(i)}\left(0 \mid \vec{z}_{0}\right)$ is the initial position of particle $i$ and $\vec{Z}\left(t \mid \vec{z}_{0}\right)$ is the configuration trajectory given the initial condition $\vec{z}_{0}$. Note that the velocity of each particle is implicitly dependent on the wave function $|\Psi(t)\rangle$, as well as the position of all the other particles through Eq. (1). Each run of an experiment corresponds to a different initial condition $\vec{z}_{0}$ sampled randomly from the initial distribution $\mathbb{P}_{0}\left(\vec{z}_{0}\right)=$ $\operatorname{Tr}\left\{\hat{\Pi}\left(\vec{z}_{0}\right) \hat{\sigma}^{(\Psi)}(0)\right\}$, which is just the Born rule. Thus, each run corresponds to a different trajectory $\vec{Z}\left(t \mid \vec{z}_{0}\right)$ and the velocity field Eq. (1) guarantees that the Born rule is obeyed at all subsequent times [44].

Now let us take particle $i=0$ as our system of interest. Hereafter, we refer to this particle as "the system" and all other particles as "the environment." The idea behind the CWF of the system is the following-given an initial configuration $\vec{z}_{0}$, the CWF generates exactly the same system trajectory that 
is generated by the combined system and environment wave function $|\Psi(t)\rangle$ from Eqs. (1) and (2). If the same trajectories are generated, then the same statistical predictions are obtained [23]. The wave function $|\Psi(t)\rangle$ lives on the Hilbert space associated with the $N$ particles and evolves according to a linear Schrödinger equation (LSE). The conditional wave function, however, lives on the reduced Hilbert space of the system only and evolves according to a NSE. Of particular interest is that the NSE contains additional terms relative to the LSE, which are directly related to entanglement. This allows us to trace back the role of entanglement in the evolution of the conditional wave function and, ultimately, in the energy fluctuations of the system.

Let us denote the position of the system by $\vec{x}:=\vec{z}^{(0)}$ and the position of the environment by $\vec{y}:=\left[\vec{z}^{(1)}, \ldots, \vec{z}^{(N-1)}\right]$, such that we can write $\vec{z}=(\vec{x}, \vec{y})$. The unnormalized CWF for the system is then defined as

$$
\begin{aligned}
\left|\tilde{\phi}^{(\Psi)}\left(t \mid \vec{z}_{0}\right)\right\rangle & =\int d \mathbf{x} \tilde{\phi}^{(\Psi)}\left(\vec{x}, t \mid \vec{z}_{0}\right)|\vec{x}\rangle \\
& =\int d \mathbf{x} \Psi\left(\vec{x}, \vec{Y}\left(t \mid \vec{z}_{0}\right), t\right)|\vec{x}\rangle,
\end{aligned}
$$

where $d \mathbf{x}$ is an infinitesimal three-dimensional volume element, $\left.\vec{Y}\left(t \mid \vec{z}_{0}\right):=\left[\vec{Z}^{(1)}\left(t \mid \vec{z}_{0}\right), \ldots, \vec{Z}^{(N-1)}\right]\left[t \mid \vec{z}_{0}\right)\right]$ is the trajectory of the environment configuration, and the position representation of $|\Psi(t)\rangle$ at the point $\vec{z}=\left(\vec{x}, \vec{Y}\left(t \mid \vec{z}_{0}\right)\right)$ is $\Psi\left(\vec{x}, \vec{Y}\left(t \mid \vec{z}_{0}\right), t\right):=\left\langle\vec{x}, \vec{Y}\left(t \mid \vec{z}_{0}\right) \mid \Psi(t)\right\rangle$. The CWF is conditioned by the trajectory of the environmental degrees of freedom and thus an explicit function of $\vec{x}$ and $t$ only. In this sense, it is a single-particle wave function. Further, it admits an operational interpretation within standard quantum mechanics in terms of weak measurements.

The weak value of an operator $\hat{O}$ with preselection on a state $|s\rangle$ and postselection on a state $\left|s^{\prime}\right\rangle$ is given by

$$
\langle\hat{O}\rangle_{s}^{s^{\prime}}:=\frac{\left\langle s^{\prime}|\hat{O}| s\right\rangle}{\left\langle s^{\prime} \mid s\right\rangle} .
$$

We now choose $|s\rangle=|\Psi(t)\rangle,\left|s^{\prime}\right\rangle=\left|\vec{p}_{S}\right\rangle \otimes\left|\vec{Y}\left(t \mid \vec{z}_{0}\right)\right\rangle$, where $\left|\vec{p}_{S}\right\rangle$ is a momentum eigenstate of the system, and $\hat{O}=$ $\hat{\Pi}^{S}(\vec{x}) \otimes \hat{1}_{E}$, where $\hat{\Pi}^{S}(\vec{x}):=|\vec{x}\rangle\langle\vec{x}|$ is a projection operator associated with the system position basis vector $|\vec{x}\rangle$ acting only on the system. Then,

$$
\begin{aligned}
\left\langle\hat{\Pi}^{S}(\vec{x}) \otimes \hat{1}_{E}\right\rangle_{\Psi}^{\vec{p}_{S} \otimes \vec{Y}} & =\frac{\left\langle\vec{p}_{S} \mid \vec{x}\right\rangle\left\langle\vec{x}, \vec{Y}\left(t \mid \vec{z}_{0}\right) \mid \Psi(t)\right\rangle}{\left\langle\vec{p}_{S}, \vec{Y}\left(t \mid \vec{z}_{0}\right) \mid \Psi(t)\right\rangle} \\
& =\frac{\exp \left\{i \vec{p}_{S} \cdot \vec{x}\right\}}{\left\langle\vec{p}_{S}, \vec{Y}\left(t \mid \vec{z}_{0}\right) \mid \Psi(t)\right\rangle} \tilde{\phi}^{(\Psi)}\left(\vec{x}, t \mid \vec{z}_{0}\right) .
\end{aligned}
$$

Choosing a zero-momentum state $\vec{p}_{S}=\overrightarrow{0}$ the prefactor in Eq. (5) does not depend on $\vec{x}$, making the weak value proportional to the value of the CWF at position $\vec{x}$. Since the trajectory of the environment can also be inferred via weak measurements, the CWF trajectories are experimentally accessible. For further details, see Ref. [43].

\section{B. Dynamics}

To derive the NSE governing the CWF evolution we look at its explicit time derivative. Taking into account the explicit time dependence of $\Psi\left(\vec{x}, \vec{Y}\left(t \mid \vec{z}_{0}\right), t\right)$ through $\vec{Y}_{t}:=\vec{Y}\left(t \mid \vec{z}_{0}\right)$, we have

$$
\begin{aligned}
\partial_{t}\left|\tilde{\phi}^{(\Psi)}\left(t \mid \vec{z}_{0}\right)\right\rangle= & \int d \mathbf{x} \partial_{t} \Psi\left(\vec{x}, \vec{Y}_{t}, t\right)|\vec{x}\rangle \\
= & -\frac{l}{\hbar} \int d \mathbf{x}\left[\left\langle\vec{x}, \vec{Y}_{t}|\hat{H}(t)| \Psi(t)\right\rangle\right. \\
& \left.-\vec{v}^{(y)} \cdot\left\langle\vec{x}, \vec{Y}_{t}\left|\hat{\vec{P}}^{(y)}\right| \Psi(t)\right\rangle\right]|\vec{x}\rangle,
\end{aligned}
$$

where we have introduced the shorthand notation $\vec{v}^{(y)} \cdot\left\langle\vec{x}, \vec{Y}_{t}\left|\hat{\vec{P}}^{(y)}\right| \Psi(t)\right\rangle:=-\imath \hbar \sum_{i=1}^{N-1} \sum_{j=1}^{3} v_{j}^{(i)}\left[\vec{Z}\left(t, \vec{z}_{0}\right), t\right]$ $\left(\partial_{y_{j}^{(i)}} \Psi\right)\left(\vec{x}, \vec{Y}_{t}, t\right)$ with $\partial_{y_{j}^{(i)}}$ the partial derivative with respect to the $j$ th component of the position of the environment particle $i$. We have used the fact that, by definition, $\quad d_{t} Y_{j}^{(i)}\left(t \mid \vec{z}_{0}\right)=v_{j}^{(i)}\left[\vec{Z}\left(t \mid \vec{z}_{0}\right), t\right]$ and that $\left(\partial_{y_{j}^{(i)}} \Psi\right)\left(\vec{x}, \vec{Y}_{t}, t\right)=(l / \hbar)\left\langle\vec{x}, \vec{Y}_{t}\left|\hat{P}_{j}^{(i)}\right| \Psi(t)\right\rangle$.

Let $\hat{H}(t)=\hat{H}_{S}(t)+\hat{H}_{E}+\hat{H}_{\text {int }}$, where $\hat{H}_{S}(t)$ is the system Hamiltonian acting only on the system degrees of freedom, $\hat{H}_{E}$ is the environment Hamiltonian acting only on the environment degrees of freedom, and $\hat{H}_{\text {int }}$ is an interaction Hamiltonian which acts on both system and environment degrees of freedom. From the Schrödinger equation $\imath \hbar \partial_{t}|\Psi(t)\rangle=$ $\hat{H}(t)|\Psi(t)\rangle$, it then follows that the evolution of the CWF in the position representation can be written in the generalized Schrödinger form

$$
\begin{aligned}
\partial_{t} \tilde{\phi}^{(\Psi)}\left(\vec{x}, t \mid \vec{z}_{0}\right) \\
=\left\langle\vec{x}, \vec{Y}_{t} \mid \partial_{t} \Psi(t)\right\rangle+\frac{l}{\hbar} \vec{v}^{(y)} \cdot\left\langle\vec{x}, \vec{Y}_{t}\left|\hat{\vec{P}}^{(y)}\right| \Psi(t)\right\rangle \\
=-\frac{l}{\hbar}\left\{\left\langle\vec{x}, \vec{Y}_{t}|\hat{H}(t)| \Psi(t)\right\rangle-\vec{v}^{(y)} \cdot\left\langle\vec{x}, \vec{Y}_{t} \mid \hat{\vec{P}}^{(y)} \Psi(t)\right\rangle\right\} \\
=-\frac{l}{\hbar}\left\{\left\langle\vec{x}\left|\hat{H}_{S}(t)\right| \tilde{\phi}\left(t \mid \vec{z}_{0}\right)\right\rangle+\left\langle\vec{x}, \vec{Y}_{t}\left|\hat{H}_{\mathrm{int}}\right| \Psi(t)\right\rangle .\right. \\
\left.+\left\langle\vec{x}, \vec{Y}_{t}\left|\hat{H}_{E}\right| \Psi(t)\right\rangle-\vec{v}^{(y)} \cdot\left\langle\vec{x}, \vec{Y}_{t}\left|\hat{\vec{P}}^{(y)}\right| \Psi(t)\right\rangle\right\} .
\end{aligned}
$$

This form is particularly interesting because we can decompose the evolution of the CWF into three distinct terms. The first term is the contribution from the system's own Hamiltonian. The second term contains the effect of the interaction term. The last two terms, perhaps the most interesting here, can be associated with entanglement between the system and environment (see Sec. III below).

Finally, for the purpose of calculations and presentation, it is more convenient to express Eqs. (6) and (7) in terms of the unnormalized density operator $\hat{\tilde{\rho}}^{(\Psi)}\left(t \mid \vec{z}_{0}\right)=$ $\left|\tilde{\phi}^{(\Psi)}\left(t \mid \vec{z}_{0}\right)\right\rangle\left\langle\tilde{\phi}^{(\Psi)}\left(t \mid \vec{z}_{0}\right)\right|$. The evolution of $\hat{\tilde{\rho}}^{(\Psi)}$ is then given by

$$
d_{t} \hat{\tilde{\rho}}^{(\Psi)}\left(t \mid \vec{z}_{0}\right)=d_{t} \hat{\tilde{\rho}}_{\mathrm{S}}^{(\Psi)}\left(t \mid \vec{z}_{0}\right)+d_{t} \hat{\tilde{\rho}}_{\mathrm{int}}^{(\Psi)}\left(t \mid \vec{z}_{0}\right)+d_{t} \hat{\tilde{\rho}}_{\mathrm{ent}}^{(\Psi)}\left(t \mid \vec{z}_{0}\right)
$$

where

$$
\begin{gathered}
d_{t} \hat{\tilde{\rho}}_{\mathrm{S}}^{(\Psi)}=-\frac{l}{\hbar}\left[\hat{H}_{S}, \hat{\tilde{\rho}}^{(\Psi)}\right], \\
d_{t} \hat{\tilde{\rho}}_{\mathrm{int}}^{(\Psi)}=-\frac{l}{\hbar}\left\langle\vec{Y}_{t}\left|\left[\hat{H}_{\mathrm{int}}, \hat{\sigma}^{(\Psi)}(t)\right]\right| \vec{Y}_{t}\right\rangle_{E}, \\
d_{t} \hat{\tilde{\rho}}_{\mathrm{ent}}^{(\Psi)}=-\frac{l}{\hbar}\left[\left\langle\vec{Y}_{t}\left|\left[\hat{H}_{E}, \hat{\sigma}^{(\Psi)}(t)\right]\right| \vec{Y}_{t}\right\rangle_{E}\right. \\
\left.-v^{(y)} \cdot\left\langle\vec{Y}_{t}\left|\left[\hat{\vec{P}}^{(y)}, \hat{\sigma}^{(\Psi)}(t)\right]\right| \vec{Y}_{t}\right\rangle_{E}\right] .
\end{gathered}
$$


We have here used the notation $\left\langle\vec{Y}_{t}|\hat{A}| \vec{Y}_{t}\right\rangle_{E}:=\operatorname{Tr}_{\mathrm{E}}\left[\hat{\Pi}^{\mathrm{E}}\left(\vec{Y}_{t}\right) \hat{A}\right]$ for any operator $\hat{A}$ in the combined Hilbert space of system and environment where $\hat{\Pi}^{E}(\vec{y}):=|\vec{y}\rangle\langle\vec{y}|$ is a projection operator similar to $\hat{\Pi}(\vec{z})$ but acting only on the environment degrees of freedom. We note that this equation is stochastic in nature despite having no explicit noise terms, because the initial conditions are stochastic (at least for the position $\vec{z}_{0}$ ). However, once $\vec{z}_{0}$ and $|\Psi(0)\rangle$ have been specified the evolution is completely deterministic.

\section{CONDITIONAL ENERGY}

To study energy fluctuations we need first to provide a link between the CWF of the system and its energy. Different proposals can be found in the literature to study fluctuations in open quantum systems [5-7,48-52]. For descriptions based on stochastic states (pure or mixed), a typical approach to estimate the energy of the system is to take the expectation value of the system Hamiltonian with respect to the stochastic state (see, e.g., Refs. [7,53]). We adopt this approach here. To this end, we introduce the concept of conditional energy as the expectation value of $\hat{H}_{S}(t)$ with respect to the CWF. Explicitly, we define the quantity

$$
\begin{aligned}
u^{(\Psi)}\left(t \mid \vec{z}_{0}\right) & :=\frac{\left\langle\tilde{\phi}^{(\Psi)}\left(t \mid \vec{z}_{0}\right)\left|\hat{H}_{S}(t)\right| \tilde{\phi}^{(\Psi)}\left(t \mid \vec{z}_{0}\right)\right\rangle}{\left\langle\tilde{\phi}^{(\Psi)}\left(t \mid \vec{z}_{0}\right) \mid \tilde{\phi}^{(\Psi)}\left(t \mid \vec{z}_{0}\right)\right\rangle} \\
& =\frac{\operatorname{Tr}\left\{\hat{\tilde{\rho}}^{(\Psi)}\left(t \mid \vec{z}_{0}\right) \hat{H}_{S}(t)\right\}}{\operatorname{Tr}\left\{\hat{\tilde{\rho}}^{(\Psi)}\left(t \mid \vec{z}_{0}\right)\right\}},
\end{aligned}
$$

as the conditional energy of the system. In analogy to the $\mathrm{CWF}$, the conditional energy is conditioned by a trajectory of the environment or, equivalently, by the initial condition $\vec{z}_{0}$. We will demonstrate below that this conditional energy provides a meaningful way to estimate the energy of the system for individual trajectories of the environment.

Note that the conditional energy is the conditional expectation value of the "Bohmian energy" introduced in Ref. [48], i.e., $u^{(\Psi)}\left(t \mid \vec{z}_{0}\right)=\mathbb{E}\left[E^{(\Psi)}(\vec{z}, t) \mid \vec{y}=\vec{Y}_{t}\right]$. In the trivial case of no environment, the conditional energy is simply the expectation value of the Bohmian energy.

To identify the different terms contributing to the fluctuations of the conditional energy we look at its total time derivative, namely

$$
\begin{aligned}
d_{t} u^{(\Psi)}\left(t \mid \vec{z}_{0}\right)= & \frac{\operatorname{Tr}\left\{\left[\hat{H}_{S}(t)-u^{(\Psi)}\left(t \mid \vec{z}_{0}\right)\right] d_{t} \hat{\tilde{\rho}}^{(\Psi)}\left(t \mid \vec{z}_{0}\right)\right\}}{\operatorname{Tr}\left\{\hat{\tilde{\rho}}^{(\Psi)}\left(t \mid \vec{z}_{0}\right)\right\}} \\
& +\frac{\operatorname{Tr}\left\{\hat{\tilde{\rho}}^{(\Psi)} d_{t} \hat{H}_{\mathrm{S}}(t)\right\}}{\operatorname{Tr}\left\{\hat{\tilde{\rho}}^{(\Psi)}\left(t \mid \vec{z}_{0}\right)\right\}}
\end{aligned}
$$

Using Eq. (8) it is straightforward to show that the conditional energy flow splits into three terms as

$$
d_{t} u^{(\Psi)}\left(t \mid \vec{z}_{0}\right)=d_{t} u_{\mathrm{ext}}^{(\Psi)}\left(t \mid \vec{z}_{0}\right)+d_{t} u_{\mathrm{int}}^{(\Psi)}\left(t \mid \vec{z}_{0}\right)+d_{t} u_{\mathrm{ent}}^{(\Psi)}\left(t \mid \vec{z}_{0}\right),
$$

where

$$
\begin{gathered}
d_{t} u_{\mathrm{ext}}^{(\Psi)}\left(t \mid \vec{z}_{0}\right)=\frac{\operatorname{Tr}\left\{\hat{\tilde{\rho}}^{(\Psi)}\left(t \mid \vec{z}_{0}\right) d_{t} \hat{H}_{\mathrm{S}}(t)\right\}}{\operatorname{Tr}\left\{\hat{\tilde{\rho}}^{(\Psi)}\left(t \mid \vec{z}_{0}\right)\right\}}, \\
d_{t} u_{\mathrm{int}}^{(\Psi)}\left(t \mid \vec{z}_{0}\right)=\frac{\operatorname{Tr}\left\{\left[\hat{H}_{S}(t)-u^{(\Psi)}\left(t \mid \vec{z}_{0}\right)\right] d_{t} \hat{\tilde{\rho}}_{\mathrm{int}}^{(\Psi)}\left(t \mid \vec{z}_{0}\right)\right\}}{\operatorname{Tr}\left\{\hat{\tilde{\rho}}^{(\Psi)}\left(t \mid \vec{z}_{0}\right)\right\}}, \\
d_{t} u_{\mathrm{ent}}^{(\Psi)}\left(t \mid \vec{z}_{0}\right)=\frac{\operatorname{Tr}\left\{\left[\hat{H}_{S}(t)-u^{(\Psi)}\left(t \mid \vec{z}_{0}\right)\right] d_{t} \hat{\tilde{\rho}}_{\mathrm{ent}}^{(\Psi)}\left(t \mid \vec{z}_{0}\right)\right\}}{\operatorname{Tr}\left\{\hat{\tilde{\rho}}^{(\Psi)}\left(t \mid z_{0}\right)\right\}} .
\end{gathered}
$$

This is our main result and allows a straightforward physical interpretation according to which the three terms can be called the external, interaction, and entanglement contributions to the rate of energy exchange, respectively. This is based on the following observations: (i) If there is no explicit time dependence in the system Hamiltonian, i.e., $d_{t} \hat{H}_{\mathrm{S}}=0$, then the first term vanishes, i.e., $d_{t} u_{\mathrm{ext}}=0$, while (ii) in the absence of interaction between the system and the environment, i.e., $\hat{H}_{\text {int }}=0$, the second term vanishes, i.e., $d_{t} u_{\text {int }}^{(\Psi)}=0$. Finally, (iii) if the system and environment are not entangled at time $t$, then the entanglement contribution to the conditional energy flow vanishes, i.e., $d_{t} u_{\mathrm{ent}}^{(\Psi)}=0$. To see this, consider a factorized state $|\Psi(t)\rangle=|\phi(t)\rangle \otimes|\chi(t)\rangle$, with $|\phi(t)\rangle$ the system wave function and $|\chi(t)\rangle$ the environmental wave function. In Eq. (11) the operators $\hat{H}_{E}$ and $\hat{\vec{P}}^{(y)}$ do not act on the system, and hence the trace over the environment leaves just a term proportional to the system state, i.e., $d_{t} \hat{\tilde{\rho}}_{\text {ent }}^{(\phi \otimes \chi)}\left(t \mid \vec{z}_{0}\right)=f\left(t \mid \vec{z}_{0}\right)|\phi(t)\rangle\langle\phi(t)|$ with $f\left(t \mid \vec{z}_{0}\right)$ a complexvalued function that depends only on time and the initial conditions $\vec{z}_{0}$. Noting that the conditional wave function reduces to $\left|\tilde{\phi}^{(\phi \otimes \chi)}\left(t \mid \vec{z}_{0}\right)\right\rangle=\left\langle\vec{Y}_{t} \mid \chi(t)\right\rangle|\phi(t)\rangle$, we see that $\operatorname{Tr}\left\{\hat{\tilde{\rho}}^{(\phi \otimes \chi)}\left(t \mid \vec{z}_{0}\right)\right\}=\left|\left\langle\vec{Y}_{t} \mid \chi(t)\right\rangle\right|^{2}$ and the conditional energy reduces to $u^{(\phi \otimes \chi)}\left(t \mid \vec{z}_{0}\right)=\left|\left\langle\vec{Y}_{t} \mid \chi(t)\right\rangle\right|^{2}\left\langle\phi(t)\left|\hat{H}_{S}(t)\right| \phi(t)\right\rangle /$ $\left|\left\langle\vec{Y}_{t} \mid \chi(t)\right\rangle\right|^{2}=\left\langle\phi(t)\left|\hat{H}_{S}(t)\right| \phi(t)\right\rangle$. Thus, if follows from Eq. (17) that the entanglement contribution vanishes as

$$
\begin{aligned}
d_{t} u_{\mathrm{ent}}^{(\phi \otimes x)}\left(t \mid \vec{z}_{0}\right)= & \frac{f\left(t \mid \vec{z}_{0}\right)}{\left|\left\langle\vec{Y}_{t} \mid \chi(t)\right\rangle\right|^{2}}\left[\left\langle\phi(t)\left|\hat{H}_{S}(t)\right| \phi(t)\right\rangle\right. \\
& \left.-u^{(\phi \otimes \chi)}\left(t \mid \vec{z}_{0}\right)\right]=0 .
\end{aligned}
$$

To make the connection to the canonical average system energy $\operatorname{Tr}\left\{\hat{H}_{S}(t) \hat{\sigma}^{(\Psi)}(t)\right\}$, we need to consider the statistical average of the conditional energy, $\left\langle\left\langle u^{(\Psi)}(t)\right\rangle\right\rangle$, over the initial configuration space points $\vec{z}_{0}$, namely

$$
\begin{aligned}
\left\langle\left\langle u^{(\Psi)}(t)\right\rangle\right\rangle & =\int d \mathbf{z}_{0}\left|\Psi\left(\vec{z}_{0}, 0\right)\right|^{2} u^{(\Psi)}\left(t \mid \vec{z}_{0}\right) \\
& =\int d \mathbf{z}_{0}\left|\Psi\left(\vec{z}_{0}, 0\right)\right|^{2}\left\langle\phi^{(\Psi)}\left(t \mid \vec{z}_{0}\right)\left|\hat{H}_{S}(t)\right| \phi^{(\Psi)}\left(t \mid \vec{z}_{0}\right)\right\rangle \\
& =\int d \mathbf{z}_{0}\left|\Psi\left(\vec{z}_{0}, 0\right)\right|^{2} \frac{\left\langle\tilde{\phi}^{(\Psi)}\left(t \mid \vec{z}_{0}\right)\left|\hat{H}_{S}(t)\right| \tilde{\phi}^{(\Psi)}\left(t \mid \vec{z}_{0}\right)\right\rangle}{\left\langle\tilde{\phi}^{(\Psi)}\left(t \mid \vec{z}_{0}\right) \mid \tilde{\phi}^{(\Psi)}\left(t \mid \vec{z}_{0}\right)\right\rangle}
\end{aligned}
$$




$$
\begin{aligned}
& =\int d \mathbf{z}_{0}\left|\Psi\left(\vec{z}_{0}, 0\right)\right|^{2} \frac{\int d \mathbf{x}\left\langle\Psi(t) \mid \vec{x}, \vec{Y}_{t}\right\rangle\left\langle\vec{x}, \vec{Y}_{t}\left|\hat{H}_{S}(t)\right| \Psi(t)\right\rangle}{\int d \mathbf{x}^{\prime}\left\langle\Psi(t) \mid \vec{x}^{\prime}, \vec{Y}_{t}\right\rangle\left\langle\vec{x}^{\prime}, \vec{Y}_{t} \mid \Psi(t)\right\rangle} \\
& =\int d \mathbf{y}\left[\int d \mathbf{x}^{\prime}\left|\Psi\left(\vec{x}^{\prime}, \vec{y}, t\right)\right|^{2}\right] \frac{\int d \mathbf{x}\langle\Psi(t) \mid \vec{x}, \vec{y}\rangle\left\langle\vec{x}, \vec{y}\left|\hat{H}_{S}(t)\right| \Psi(t)\right\rangle}{\int d \mathbf{x}^{\prime}\left|\Psi\left(\vec{x}^{\prime}, \vec{y}, t\right)\right|^{2}} \\
& =\int d \mathbf{x} d \mathbf{y}\langle\Psi(t) \mid \vec{x}, \vec{y}\rangle\left\langle\vec{x}, \vec{y}\left|\hat{H}_{S}(t)\right| \Psi(t)\right\rangle \\
& =\left\langle\Psi(t)\left|\hat{H}_{S}(t)\right| \Psi(t)\right\rangle=\operatorname{Tr}\left\{\hat{H}_{S}(t) \hat{\sigma}^{(\Psi)}(t)\right\},
\end{aligned}
$$

where we have used the equivariance property $\int d \mathbf{z}_{0}$ $\left|\Psi\left(\vec{z}_{0}, 0\right)\right|^{2} f\left(Z^{(\Psi)}\left(t, \vec{z}_{0}\right), t\right)=\int d \mathbf{z}|\Psi(\vec{z}, t)|^{2} f(\vec{z}, t)$, valid for any $L^{2}$ function $f$, and $d \mathbf{z}=d \mathbf{x} d \mathbf{y}$, where $d \mathbf{y}$ is an infinitesimal volume in the configuration space of the environment. This shows that the statistical average of the conditional energy over the initial configurations indeed gives the physically meaningful expectation value of the system Hamiltonian $\hat{H}_{S}(t)$.

Furthermore, we can relate the statistical average of the conditional energy flow [Eqs. (14) to (17)] to the average energy flow $d_{t}\left\langle\Psi(t)\left|\hat{H}_{S}(t)\right| \Psi(t)\right\rangle=\operatorname{Tr}\left\{\hat{\sigma}^{(\Psi)}(t) d_{t} \hat{H}_{S}(t)\right\}+$ $\operatorname{Tr}\left\{\hat{H}_{S}(t) d_{t} \hat{\sigma}^{(\Psi)}(t)\right\}$. For the first term, we find

$$
\operatorname{Tr}\left\{\hat{\sigma}^{(\Psi)}(t) d_{t} \hat{H}_{S}(t)\right\}=\left\langle\left\langle d_{t} u_{\mathrm{ext}}^{(\Psi)}(t)\right\rangle,\right.
$$

which nicely confirms that energy exchange of the system that results from time dependence of the Hamiltonian is given by our external contribution $\left\langle\left\langle d_{t} u_{\mathrm{ext}}^{(\Psi)}(t)\right\rangle\right\rangle$ to the total conditional energy.

From Eqs. (19) and (20) it follows that

$$
\operatorname{Tr}\left\{\hat{H}_{S}(t) d_{t} \hat{\sigma}^{(\Psi)}(t)\right\}=\left\langle\left\langle d_{t} u_{\text {int }}^{(\Psi)}(t)\right\rangle\right\rangle+\left\langle\left\langle d_{t} u_{\mathrm{ent}}^{(\Psi)}(t)\right\rangle\right\rangle,
$$

and, in particular, we can show that

$$
\left\langle\left\langle d_{t} u_{\mathrm{ent}}^{(\Psi)}(t)\right\rangle=-\frac{l}{\hbar} \operatorname{Tr}\left\{\hat{\sigma}^{(\Psi)}(t)\left[\hat{u}^{(\Psi)}(t), \hat{H}_{\mathrm{int}}\right]\right\},\right.
$$

where $\hat{u}^{(\Psi)}(t)$ is a state-dependent operator acting only on the environment degrees of freedom with matrix elements $\left\langle i\left|\hat{u}^{(\Psi)}(t)\right| j\right\rangle=\int d \mathbf{y}\langle i \mid \vec{y}\rangle\langle\vec{y} \mid j\rangle u^{(\Psi)}(t, \vec{y})$ and $u^{(\Psi)}(t, \vec{y})$ is the conditional energy of the system at time $t$ for an environment configuration $\vec{y}$ at the same time. Equation (22) shows that if no interaction is present or the interaction Hamiltonian commutes with $\hat{u}^{(\Psi)}(t)$, then entanglement makes no direct contribution to the average energy change. This is in accordance with the expectation that on average no energy transfer can come about from entanglement alone, i.e, an interaction with the environment is required. We give an example below using a momentum-momentum interaction where this term is relevant.

We conclude this section with two remarks. First, the energy exchange contributions identified here are valid for any environment and interaction Hamiltonians, including timedependent interactions. In particular, no assumption is made about the structure of the environment and the form or strength of the interaction. We note that we have analyzed energy fluctuations associated with the bare system Hamiltonian $\hat{H}_{S}(t)$, i.e., the fluctuations of the term $u^{(\Psi)}\left(t \mid \vec{z}_{0}\right)=$ $\left\langle\phi^{(\Psi)}\left(t \mid \vec{z}_{0}\right)\left|\hat{H}_{S}(t)\right| \phi^{(\Psi)}\left(t \mid \vec{z}_{0}\right)\right\rangle$. When the system-environment interaction is strong, alternative system Hamiltonians have been investigated, especially in the thermodynamic setting [54-60], which attribute part of the interaction energy to the system energy. Additional energetic fluctuations could arise in this effective picture due to the difference between the bare and effective Hamiltonians.

Second, it is important to note that in deriving the results in this section we have assumed that the state $|\Psi(t)\rangle$ is known. In many experimental scenarios, the system is prepared in some well-known state before each run of a given drive protocol and if this state is known, quantities conditioned on the individual pure states are empirically accessible. However, if one only has access to the averages described through the relevant density operator $\hat{\sigma}$ representing a mixed state, then all measurable quantities have to be expressed at this level and they cannot depend on an individual decomposition of $\hat{\sigma}$. If they did, then one could distinguish between different decomposition of $\hat{\sigma}$ without a priori knowledge of its preparation, in contradiction with the predictions of quantum mechanics. We show in the Appendix how Eqs. (14) to (22) can be coarse grained to describe general mixed states based on Refs. [43,61]. The main result remains unaltered, with the only difference that the entanglement contribution should now be considered as a correlations contribution, since it will be nonzero for both quantum and classical correlations.

\section{EXAMPLES}

In this section we explicitly solve a series of simple examples to show how CWFs can be used to characterize energy flow in open quantum systems beyond the usual expectation values of the Hamiltonian. Through four examples, we illustrate different cases of interplay between external drive, interaction, and entanglement. In example $\mathrm{A}$, we present the trivial case of an externally driven system with no interaction and no entanglement with an environment. This is a check on the formalism to show that, in this case, the CWF reduces to the usual system wave function and the conditional energy to the usual expectation value of the Hamiltonian. Example B presents the case where the system and its environment can interact, but this interaction generates negligible entanglement. The standard example of this is that of a qubit interacting with an external electromagnetic field in a high energy coherent state. Example $\mathrm{C}$ presents the case where both interaction and entanglement are present. Finally, Example D presents the case where only entanglement is present. This situation can occur when the system interacted with the environment in the past and the two became entangled due to this finite interaction term $H_{\text {int }} \neq 0$. After the generation of entanglement the 
interaction can be turned off at $t=0$. Thus at $t>0$ the state of the system and the environment will remain entangled while there is no interaction term present.

\section{A. Driven system without interaction or entanglement}

The simplest example is that of no interaction $\hat{H}_{\text {int }}=0$ and no entanglement between the system and the environment. For this case the only contribution to energy change should come from the external drive term $d_{t} u_{\mathrm{ext}}^{(\Psi)}$. We can then write the total system state as a product state between the system $\hat{\rho}$ and the environment $\hat{\chi}$ as $\hat{\sigma}^{(\Psi)}(t)=\hat{\rho}(t) \otimes \hat{\chi}(t)$. Noting that $d_{t} \hat{\tilde{\rho}}_{\text {ent }}^{(\Psi)} \propto \hat{\rho}(t)$, we see that $d_{t} u_{\text {ent }}^{(\Psi)} \propto \operatorname{Tr}\left\{\hat{\rho} \hat{H}_{S}\right\}-u^{(\Psi)}=0$. Thus, as expected, the total rate of energy change is given by $d_{t} u^{(\Psi)}=d_{t} u_{\mathrm{ext}}^{(\Psi)}$.

\section{B. Interacting system with negligible entanglement}

Next, we consider the case of a driven interacting system with negligible entanglement with its environment such that $|\Psi(t)\rangle \approx|\phi(t)\rangle \otimes|\chi(t)\rangle$ for all times $t$. As an example, consider the case where the interaction has negligible effect on the state of the environment. Let the total system be the product state $\hat{\sigma}^{(\Psi)}(t)=\hat{\rho}(t) \otimes \hat{\chi}(t)$, where $\rho(t)=|\phi(t)\rangle\langle\phi(t)|$ and $\hat{\chi}(t)=|\chi(t)\rangle\langle\chi(t)|$, and an interaction Hamiltonian of the form $\hat{H}_{\mathrm{int}}(t)=\sum_{i} \hat{A}_{i}(t) \otimes \hat{B}_{i}(t)$. Then

$$
\begin{aligned}
d_{t} \hat{\tilde{\rho}}_{\text {int }}^{(\Psi)}\left(t \mid \vec{Z}_{0}\right)= & -\frac{l}{\hbar}\left\langle\vec{Y}_{t}\left|\left[\hat{H}_{\text {int }}, \hat{\sigma}^{(\Psi)}(t)\right]\right| \vec{Y}_{t}\right\rangle_{E} \\
= & -\frac{l}{\hbar} \sum_{i}\left[\hat{A}_{i} \hat{\rho}(t)\left\langle\vec{Y}_{t}\left|\hat{B}_{i} \hat{\chi}(t)\right| \vec{Y}_{t}\right\rangle_{E}\right. \\
& \left.-\hat{\rho}(t) \hat{A}_{i}\left\langle\vec{Y}_{t}\left|\hat{\chi}(t) \hat{B}_{i}\right| \vec{Y}_{t}\right\rangle_{E}\right] .
\end{aligned}
$$

Assume now that for a particular choice of operators $\hat{B}_{i}$ and environment state $\hat{\chi}$, we have $\left\langle\vec{Y}_{t}\left|\hat{B}_{i} \hat{\chi}\right| \vec{Y}_{t}\right\rangle_{E} \approx\left\langle\vec{Y}_{t}\left|\hat{\chi} \hat{B}_{i}\right| \vec{Y}_{t}\right\rangle_{E}:=$ $b_{i}\left(t \mid \vec{z}_{0}\right) \forall i, Y_{t}$. Then $d_{t} \hat{\tilde{\rho}}_{\text {int }} \approx-(l / \hbar) \sum_{i} b_{i}\left(t \mid \vec{z}_{0}\right)\left[\hat{A}_{i}(t), \hat{\rho}(t)\right]$ and the state remains factorized. In particular,

$$
|\phi(t+\delta t)\rangle=|\phi(t)\rangle-\frac{\imath \delta t}{\hbar}\left[\hat{H}_{S}(t)+\sum_{i} b_{i}\left(t \mid \vec{z}_{0}\right) \hat{A}_{i}\right]|\phi(t)\rangle,
$$

to first order in $\delta t$. Thus, the system is effectively driven by an additional term $\hat{H}_{D}\left(t \mid \vec{z}_{0}\right) \equiv \sum_{i} b_{i}\left(t \mid \vec{z}_{0}\right) \hat{A}_{i}(t)$.

Similarly to the previous subsection the entanglement contribution vanishes since $d_{t} \hat{\tilde{\rho}}_{\text {ent }}^{(\Psi)} \propto \hat{\rho}(t)$. For the interaction term we have

$$
\begin{aligned}
d_{t} u_{\mathrm{int}}^{(\Psi)} & =-\frac{l}{\hbar} \operatorname{Tr}\left\{\sum_{i} b_{i}\left(t \mid \vec{z}_{0}\right)\left[\hat{A}_{i}(t), \hat{\rho}(t)\right]\left[\hat{H}_{S}(t)-u^{(\Psi)}\left(t \mid Y_{t}\right)\right]\right\} \\
& =-\frac{l}{\hbar} \operatorname{Tr}\left\{\hat{\rho}(t)\left[\hat{H}_{S}(t), \sum_{i} b_{i}\left(t \mid \vec{z}_{0}\right) \hat{A}_{i}(t)\right]\right\} \\
& =-\frac{l}{\hbar} \operatorname{Tr}\left\{\hat{\rho}(t)\left[\hat{H}_{S}(t), \hat{H}_{D}\left(t \mid \vec{z}_{0}\right)\right]\right\} .
\end{aligned}
$$

A concrete example here is that of a qubit driven by a laser. This is described by an interaction with a harmonic oscillator in a coherent state $|\alpha\rangle\left(|\alpha|^{2} \gg 1\right)$ with an interaction Hamiltonian (neglecting fast oscillating terms) $\hat{H}_{\text {int }}=$ $\lambda_{\mathrm{c}} \exp (i \omega t) \hat{a}^{\dagger} \hat{\sigma}^{-}+\lambda_{\mathrm{c}}^{*} \exp (-i \omega t) \hat{a} \hat{\sigma}^{+}$, where $\omega$ is the natural

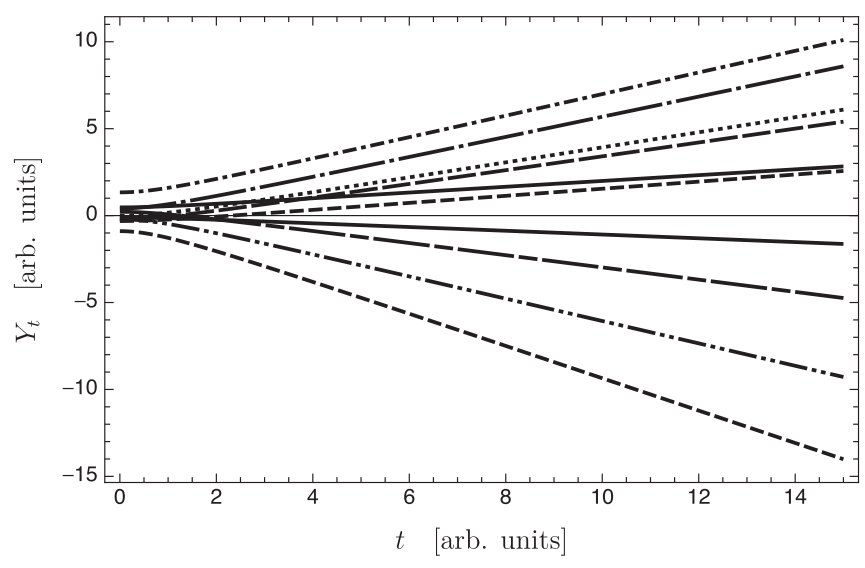

FIG. 1. Typical trajectories $Y_{t}$ for the case of two particles interacting through a quadratic potential in Sec. IVC with initial conditions randomly sampled from the probability distribution $|\Psi(x, y, 0)|^{2}$. Different dashing style indicates different initial conditions.

frequency of the qubit, $\lambda_{\mathrm{c}}$ is a coupling constant, $\hat{a}\left(\hat{a}^{\dagger}\right)$ the annihilation (creation) operator, and $\hat{\sigma}^{-}\left(\hat{\sigma}^{+}\right)$the lowering (raising) operator. Let $\hat{\sigma}^{(\Psi)}(t)=\hat{\alpha} \otimes \hat{\rho}$ be the initial state where $\hat{\alpha}$ is the density operator of a coherent state $|\alpha\rangle$. Making the approximation that $\hat{a}^{\dagger}|\alpha\rangle \approx \alpha^{*}|\alpha\rangle$ leads to $\hat{H}_{D}=\left(c \hat{\sigma}^{-}+\right.$ $\left.c^{*} \hat{\sigma}^{+}\right)$in Eq. (25), where $c=\lambda_{\mathrm{c}} \alpha \exp (i \omega t)$. Thus,

$$
d_{t} u_{\mathrm{int}}^{\mathrm{laser}}=-\frac{l}{\hbar} \operatorname{Tr}\left\{\hat{\rho}(t)\left[\hat{H}_{S}(t), c \hat{\sigma}^{-}+c^{*} \hat{\sigma}^{+}\right]\right\}
$$

as expected. Note that if we were to include the laser interaction as part of the system energy, then this contribution would appear as an explicit time-dependent term.

\section{Interaction and entanglement}

Here we consider two different cases where both interaction and entanglement contributions are present.

\section{1. $\hat{X} \otimes \hat{Y}$ interaction}

For the sake of simplicity we consider here the case of two particles interacting through a quadratic potential. The Hamiltonian in units of $m=\hbar=1$ is given by

$$
\hat{H}=\frac{1}{2}\left(\hat{P}_{X}^{2}+\hat{P}_{Y}^{2}\right)+\frac{1}{4}(\hat{X}-\hat{Y})^{2},
$$

and we start from an initial factorized state $\Psi(x, y, 0)=$ $\pi^{-1 / 2} \exp \left[-\left(x^{2}+y^{2}\right) / 2\right]$. Solving the Schrödinger equation we explicitly find that the full wave function evolves as

$$
\Psi(x, y, t)=\frac{1}{\sqrt{\pi(1+l t)}} e^{-\frac{1}{4}\left[(x+y)^{2}+(x-y)^{2} /(1+u t)+2 t t\right]},
$$

and for the trajectories we get

$$
Y_{t}:=Y\left(t \mid x_{0}, y_{0}\right)=b(t) x_{0}+a(t) y_{0},
$$

with $a(t)=\left(\sqrt{1+t^{2}}+1\right) / 2$ and $b(t)=\left(\sqrt{1+t^{2}}-1\right) / 2$. Since there are only two particles in one dimension we will drop the indices $i$ and $j$ and use the notation $x_{0}$ and $y_{0}$ to denote the initial conditions of the $X$ and $Y$ particles. Figure 1 shows some of the environment trajectories with initial conditions sampled randomly from the probability distribution 
$|\Psi(x, y, 0)|^{2}$. The unnormalized CWF is explicitly given by

$$
\begin{aligned}
& \tilde{\phi}\left(x, t \mid x_{0}, y_{0}\right) \\
& \quad=\frac{\exp -2 \imath t}{\sqrt{\pi(1+\imath t)}} e^{-\frac{1}{4}\left\{\left[x+b(t) x_{0}+a(t) y_{0}\right]^{2}+\left[x-b(t) x_{0}-a(t) y_{0}\right]^{2} /(1+t t)\right\}} .
\end{aligned}
$$

We take the system Hamiltonian to be $\hat{H}_{S}=\hat{P}_{X}^{2} / 2+\hat{X}^{2} / 4$ and the interaction Hamiltonian $\hat{H}_{\text {int }}=-\hat{X} \hat{Y} / 2$. The conditional energy and its time derivative can be readily obtained as

$$
u^{(\Psi)}\left(t \mid x_{0}, y_{0}\right)=\frac{3}{8}+\frac{t^{2} Y_{t}^{2}}{4 t^{2}+8},
$$

and

$$
d_{t} u^{(\Psi)}\left(t \mid x_{0}, y_{0}\right)=\frac{t Y_{t}\left[t\left(t^{2}+2\right) d_{t} Y_{t}+2 Y_{t}\right]}{2\left(t^{2}+2\right)^{2}} .
$$

Similarly, the interaction component of $d_{t} u$ is given by inserting Eq. (30) in Eq. (16) which yields

$$
d_{t} u_{\mathrm{int}}^{(\Psi)}\left(t \mid x_{0}, y_{0}\right)=\frac{t Y_{t}^{2}}{2\left(2+t^{2}\right)},
$$

and the entanglement contribution is then determined by $d_{t} u_{\mathrm{ent}}=d_{t} u-d_{t} u_{\mathrm{int}}$, which yields

$$
d_{t} u_{\mathrm{ent}}^{(\Psi)}\left(t \mid x_{0}, y_{0}\right)=\frac{t^{2} Y_{t}\left[\left(t^{2}+2\right) d_{t} Y_{t}-t Y_{t}\right]}{2\left(t^{2}+2\right)^{2}}
$$

The total contributions from the interaction and entanglement can be written as

$$
\begin{aligned}
\Delta u_{\mathrm{int}}^{(\Psi)}\left(t \mid x_{0}, y_{0}\right) & \\
= & \int_{0}^{t} d_{t} u_{\mathrm{int}}^{(\Psi)}\left(t \mid \vec{z}_{0}\right) d t=\frac{1}{16}\left[4\left(x_{0}^{2}-y_{0}^{2}\right) \arctan \sqrt{t^{2}+1}\right. \\
& \left.-\left(x_{0}+y_{0}\right)\left[c(t) x_{0}-d(t) y_{0}\right]+4 x_{0} y_{0} \log \frac{2}{t^{2}+2}\right] ;
\end{aligned}
$$

$$
\Delta u_{\mathrm{ent}}^{(\Psi)}\left(t \mid x_{0}, y_{0}\right)=\Delta u^{(\Psi)}\left(t \mid x_{0}, y_{0}\right)-\Delta u_{\mathrm{int}}^{(\Psi)}\left(t \mid x_{0}, y_{0}\right),
$$

respectively, where $c(t)=-t^{2}+4 \sqrt{t^{2}+1}+\pi-4, d(t)=$ $t^{2}+4 \sqrt{t^{2}+1}+\pi-4$, and $\Delta u^{(\Psi)}\left(t \mid x_{0}, y_{0}\right)=u^{(\Psi)}\left(t \mid x_{0}, y_{0}\right)-$ $u^{(\Psi)}\left(0 \mid x_{0}, y_{0}\right)$. Figure 2 shows the evolution of $\Delta u_{\text {int }}$ and $\Delta u_{\text {ent }}$ for the trajectories $Y_{t}$ in Fig. 1.

As advertised in Sec. III, the average energy flow coming from the entanglement vanishes, i.e., $\left\langle\left\langle\Delta u_{\text {ent }}^{(\Psi)}\right\rangle\right\rangle=0$ and $\left\langle\left\langle\Delta u^{(\Psi)}\right\rangle\right\rangle=\left\langle\left\langle\Delta u_{\text {int }}^{(\Psi)}\right\rangle\right\rangle=t^{2} / 16$. However, higher moments of the entanglement contribution do not vanish as evidenced by Fig. 2(b). To give an estimate of how much the entanglement fluctuations contribute to the conditional energy fluctuations, we can decompose the variance of the conditional energy into its entanglement and interaction contributions, namely

$$
\begin{aligned}
\operatorname{Var}\left\{\Delta u^{(\Psi)}\right\}= & \operatorname{Var}\left\{\Delta u_{\text {int }}^{(\Psi)}\right\}+\operatorname{Var}\left\{\Delta u_{\mathrm{ent}}^{(\Psi)}\right\} \\
& +2 \operatorname{Cov}\left\{\Delta u_{\text {int }}^{(\Psi)}, \Delta u_{\mathrm{ent}}^{(\Psi)}\right\},
\end{aligned}
$$

where $\operatorname{Cov}\{z, w\}=\langle\langle z w\rangle\rangle-\langle\langle z\rangle\rangle\langle\langle w\rangle\rangle$ is the covariance of $z$ and $w$ and $\operatorname{Var}\{z\}=\operatorname{Cov}\{z, z\}$ is the variance of $z$. Figure 3 shows these quantities as a function of time. As already seen
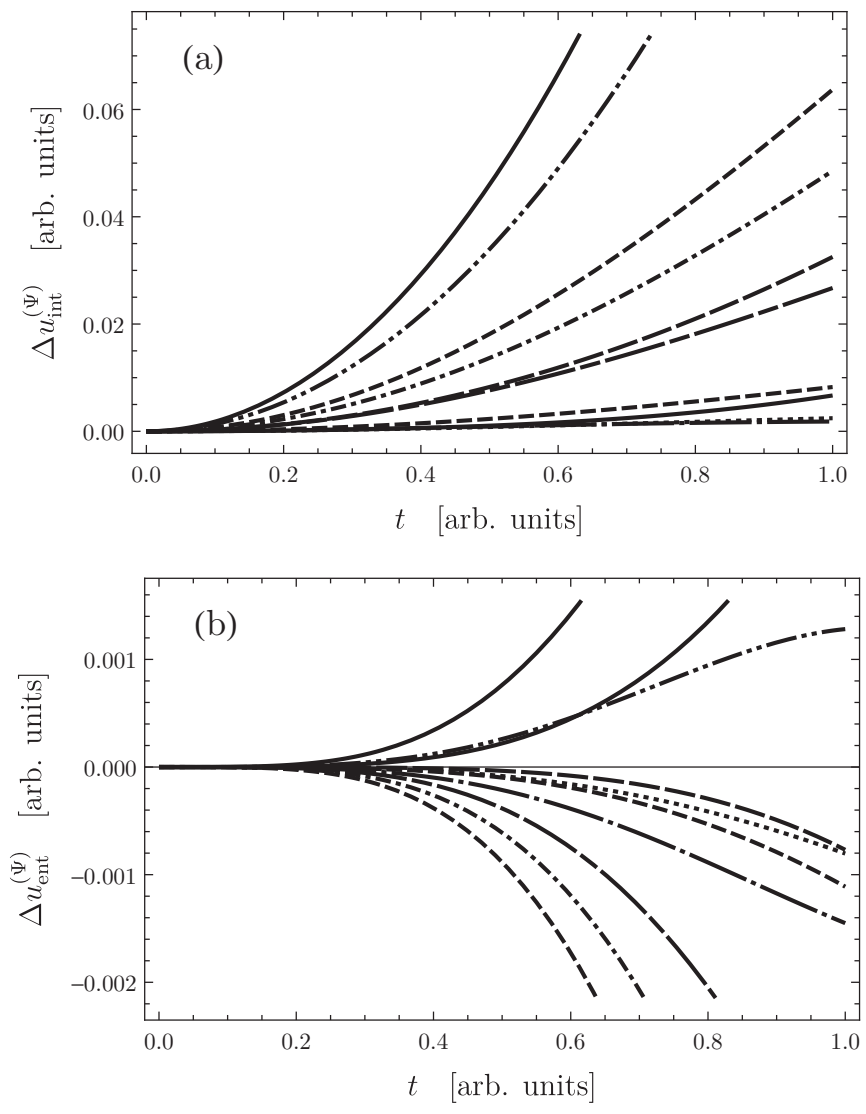

FIG. 2. Time evolution of the energy components (a) $\Delta u_{\text {int }}$ and (b) $\Delta u_{\text {ent }}$ corresponding to the trajectories $Y_{t}$ in Fig. 1. Different dashing style indicates different initial conditions as in Fig. 1.

from Fig. 2, the interaction contribution quickly dominates over the entanglement one.

\section{2. $\hat{\boldsymbol{P}}_{X} \otimes \hat{\boldsymbol{P}}_{Y}$ interaction}

We now present a case where there is an interaction term that can generate an entanglement contribution, as described in Eq. (22). As explained in Sec. III entanglement is generated

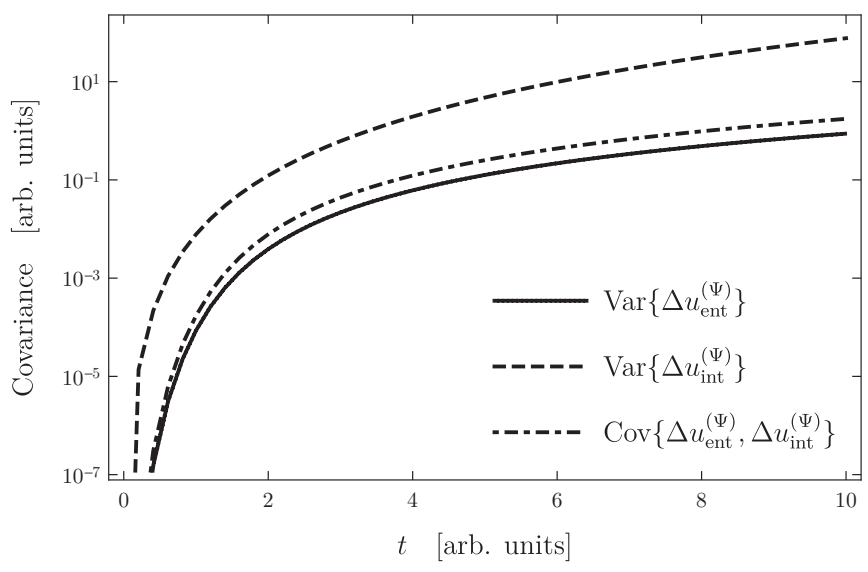

FIG. 3. Variance of $\Delta u$ decomposed into its entanglement and interaction contributions on a semilogarithmic scale (the first point is taken at $t=0.01)$. See text for details. 
if the interaction term does not commute with $\hat{u}^{(\Psi)}(t)$. We consider the interaction

$$
\hat{H}_{\text {int }}=-\lambda \hat{P}_{X} \otimes \hat{P}_{Y},
$$

for some real-valued coupling constant $\lambda$ and take $\hat{H}_{S}=\hat{P}_{X}^{2} / 2$ and $\hat{H}_{E}=\hat{P}_{Y}^{2} / 2$. This case is very similar to the previous one but now, as we will demonstrate, there is a nonzero entanglement contribution to the average energy flow. To further simplify the analysis we assume $\lambda \gg 1$ such that the interaction term dominates over the system and environment Hamiltonians and the evolution is dictated by $\hat{H}_{\text {int }}$. Again we assume an initial factorized state as in the previous case. The solution of the Schrödinger equation is then given by

$$
\Psi(x, y, t)=\frac{1}{\sqrt{\pi f(t)}} e^{-\left(x^{2}+y^{2}-2 \imath \lambda x y t\right) / 2 f(t)},
$$

with $f(t)=1+\lambda^{2} t^{2}$. Rather than solving for the trajectories we will focus on the expectation value of $\hat{H}_{S}$. A quick calculation shows that $\left\langle\Psi(t)\left|\hat{H}_{S}\right| \Psi(t)\right\rangle=\frac{1}{4}$. As expected, the average energy is constant since $\hat{H}_{S}$ commutes with $\hat{H}_{\text {int }}$. To evaluate the entanglement contribution from Eq. (22) we need the conditional energy at time $t$ given a configuration $y$ of the environment, $u^{(\Psi)}(t, y)$, and the term $\left\langle x\left|d_{t} \hat{\tilde{\rho}}_{\text {int }}\right| x\right\rangle$. The former is easily evaluated as before and gives

$$
u^{(\Psi)}(t, y)=\frac{\lambda^{2} t^{2}\left(2 y^{2}+1\right)+1}{4 f^{2}(t)} .
$$

The latter can be written in the form

$$
\begin{aligned}
\left\langle x\left|d_{t} \hat{\tilde{\rho}}_{\text {int }}\right| x\right\rangle & =-\imath\left\langle y, x\left|\left[\hat{H}_{\text {int }}, \hat{\sigma}^{(\Psi)}\right]\right| x, y\right\rangle \\
& =\imath\left(\Psi^{*} \partial_{x} \partial_{y} \Psi-\Psi \partial_{x} \partial_{y} \Psi^{*}\right) \\
& =-2 \operatorname{Im}\left(\Psi^{*} \partial_{x} \partial_{y} \Psi\right) \\
& =\frac{2 \lambda t}{\pi f^{3}(t)} e^{-\left(x^{2}+y^{2}\right) / f(t)}\left[x^{2}+y^{2}-f(t)\right] .
\end{aligned}
$$

Finally, plugging Eqs. (40) and (41) into Eq. (22) and performing the integration we find

$$
\left\langle\left\langle d_{t} u_{\mathrm{ent}}^{(\Psi)}(t)\right\rangle=\frac{\lambda^{3} t^{3}}{2 f^{2}(t)} .\right.
$$

Since $\left\langle\left\langle u^{(\Psi)}(t)\right\rangle\right\rangle$ is constant and there are no explicit time-dependent terms in the Hamiltonian, it follows that $\left\langle\left\langle d_{t} u_{\text {int }}^{(\Psi)}(t)\right\rangle\right\rangle=-\left\langle\left\langle d_{t} u_{\text {ent }}^{(\Psi)}(t)\right\rangle\right\rangle$. The entanglement and interaction flow contributions to the expectation value of $\hat{H}_{S}$ are plotted in Fig. 4.

\section{Initial entanglement but no further interaction}

We now consider the case that previous interaction between the system and the environment generated entanglement, but the interaction has been turned off at $t=0$, i.e., $\hat{H}_{\text {int }}=0$. As an example, we consider two spin $1 / 2$ particles initially in the entangled state

$$
\Psi(x, y, 0)=\frac{1}{\sqrt{2}}\left[\begin{array}{c}
g(y) e^{\imath k x} f(x) \\
g(y) f(x) \\
0 \\
0
\end{array}\right],
$$

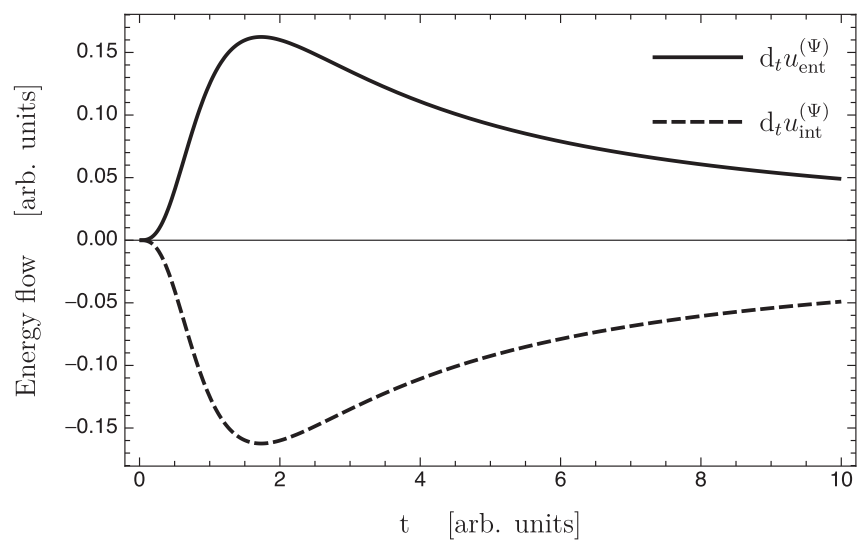

FIG. 4. Entanglement and interaction flow contributions to the expectation value of $\hat{H}_{S}$ for the example in Sec. IV C 2.

where $f(x)=\left(2 \pi \sigma_{X}^{2}\right)^{-1 / 4} \exp \left(-x^{2} / 4 \sigma_{X}^{2}\right)$ and $g(y)=$ $\left(2 \pi \sigma_{Y}^{2}\right)^{-1 / 4} \exp \left(-y^{2} / 4 \sigma_{Y}^{2}\right) \quad$ with $\quad \sigma_{X}, \sigma_{Y}>0$, and the spin components are represented in the basis $\left\{\left|\uparrow_{Y}\right\rangle\left|\uparrow_{X}\right\rangle,\left|\downarrow_{Y}\right\rangle\left|\downarrow_{X}\right\rangle,\left|\uparrow_{Y}\right\rangle\left|\downarrow_{X}\right\rangle,\left|\downarrow_{Y}\right\rangle\left|\uparrow_{X}\right\rangle\right\}$. A unitary rotation $\hat{U}_{Y}(t)=\exp \left(-l v t \hat{P}_{Y}\left|\uparrow_{Y}\right\rangle\left\langle\uparrow_{Y}\right|\right) \otimes \mathbb{1}_{X}$ acting only on particle $Y$ is then applied, where $v$ is a real-valued constant, $t$ is the duration of the unitary, and $\hat{P}_{Y}$ is the momentum operator of particle $Y$. We assume that $t$ is short enough that the free evolution contribution from the individual particles can be ignored. The resulting state is given by

$$
\Psi(x, y, t)=\frac{1}{\sqrt{2}}\left[\begin{array}{c}
g(y-v t) e^{i k x} f(x) \\
g(y) f(x) \\
0 \\
0
\end{array}\right] .
$$

For large-enough $v t$, the unitary $\hat{U}_{Y}$ separates the wave function into two wave packets with disjoint $y$ support, one centered at $(x, y)=(0,0)$ and another at $(x, y)=(0, v t)$. Thus, at the end only one of the terms in Eq. (44) is relevant for the conditional wave function since either $g\left(Y_{t}\right) \approx 0$ or $g\left(Y_{t}-v t\right) \approx 0$. During the rotation $\hat{U}_{Y}$ no interaction term exists between the particles and the Hamiltonian of particle $Y$ has no explicit time dependence. Thus, the only contribution comes from entanglement. To get the trajectories we use the continuity equation. The probability distribution over the configuration space, $\mu(x, y, t):=|\Psi(x, y, t)|^{2}$, is given by summing the distributions associated with the individual spin components [23]. In this case, $\mu(x, y, t)=$ $\sum_{s \in\{\uparrow, \downarrow\}} \sum_{s^{\prime} \in\{\uparrow, \downarrow\}} \Psi_{s, s^{\prime}}^{*}(x, y, t) \Psi_{s, s^{\prime}}(x, y, t)=g^{2}(y+v t) f^{2}$ $(x) / 2+g^{2}(y) f^{2}(x) / 2$, where $\Psi_{s, s^{\prime}}(x, y, t)$ is the $\left|s_{X}\right\rangle\left|s_{Y}^{\prime}\right\rangle$ component of $\Psi$. Thus,

$$
\begin{aligned}
\partial_{t} \mu(x, y, t) & =-v g(y+v t)\left[\partial_{y} g^{2}(y+v t)\right] f^{2}(x) \\
& =\partial_{y}\left[v \frac{1}{2} g^{2}(y+v t) f^{2}(x)\right] \\
& =-\partial_{y}\left[\frac{v \mu_{\uparrow \uparrow}(x, y, t)}{\mu(x, y, t)} \mu(x, y, t)\right] \\
& =-\partial_{y}\left[v_{y}(y, t) \mu(x, y, t)\right],
\end{aligned}
$$

where $\quad \mu_{\uparrow \uparrow}(x, y, t)=g^{2}(y+v t) f^{2}(x) / 2 \quad$ and $\quad v_{y}(y, t)=$ $v g^{2}(y+v t) /\left[g^{2}(y+v t)+g^{2}(y)\right]$. With the condition that 

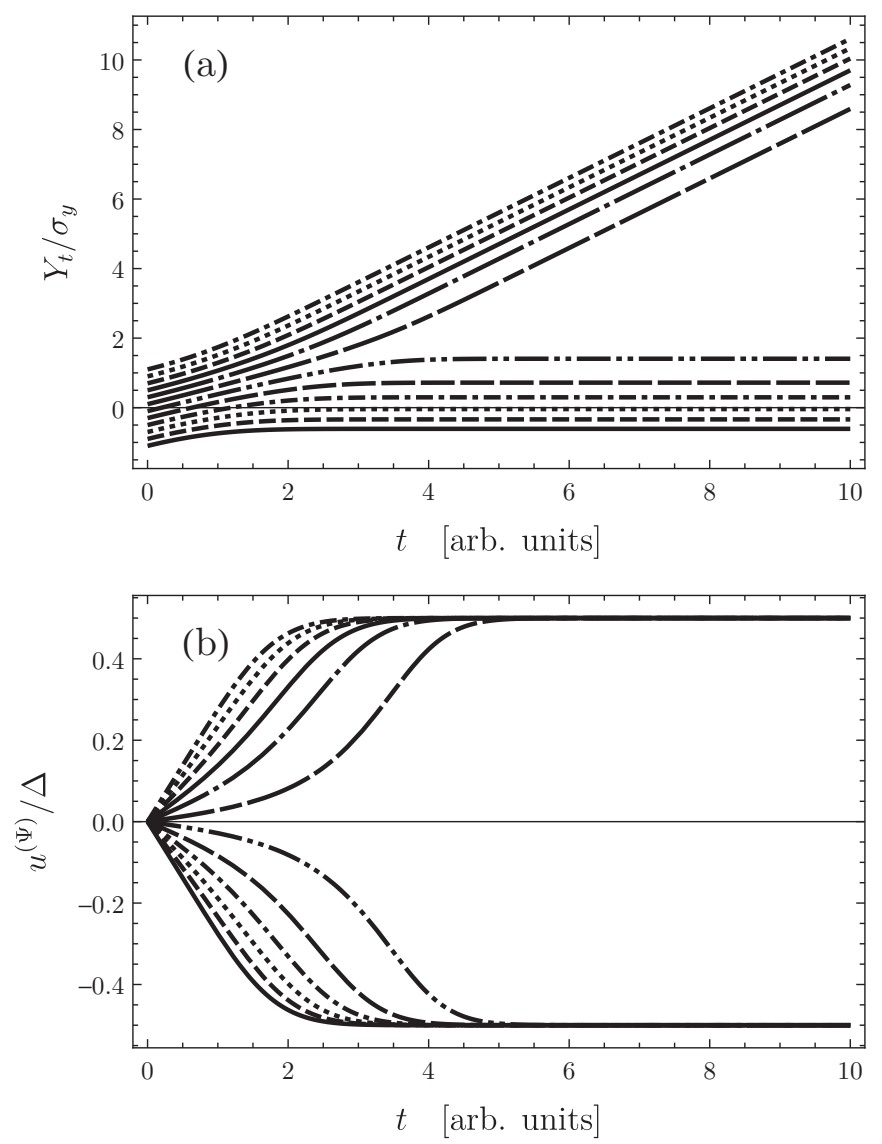

FIG. 5. (a) Particle trajectories $Y_{t}$ (normalized with the standard deviation) for evenly separated initial positions for the case of two entangled spin-1/2 particles. (b) The conditional energy change corresponding to the trajectories in (a) normalized with the splitting parameter $\Delta=\hbar^{2} k^{2} /(2 m)$.

the probability current density vanishes at infinity, this implies that the velocity field for the $Y$ particle is given by $v_{y}(y, t)$. Typical trajectories for the $Y$ particle are plotted in Fig. 5(a) for different initial conditions.

The conditional energy is evaluated for the kinetic energy of particle $X$, since no other potential is present, which yields

$$
\begin{aligned}
u^{(\Psi)}\left(t \mid \vec{z}_{0}\right) & \\
= & -\frac{\hbar^{2}}{2 m} \frac{\int d x \sum_{s \in\{\uparrow, \downarrow\}} \sum_{s^{\prime} \in\{\uparrow, \downarrow\}} \Psi_{s, s^{\prime}}^{*}\left(x, Y_{t}, t\right) \partial_{x}^{2} \Psi_{s, s^{\prime}}\left(x, Y_{t}, t\right)}{\int d x \mu\left(x, Y_{t}, t\right)} \\
= & -\left[\frac{\hbar^{2} g^{2}\left(Y_{t}-v t\right)}{4 m} \int d x e^{l k x} f(x) \partial_{x}^{2}\left[e^{-l k x} f(x)\right]\right. \\
& \left.+\frac{\hbar^{2} g^{2}\left(Y_{t}\right)}{4 m} \int d x f(x) \partial_{x}^{2} f(x)\right] \frac{1}{\int d x \mu\left(x, Y_{t}, t\right)} \\
= & \frac{\hbar^{2}}{2 m}\left[\frac{1}{4 \sigma_{X}^{2}}+k^{2} \frac{g^{2}\left(Y_{t}-v t\right)}{g^{2}\left(Y_{t}-v t\right)+g^{2}\left(Y_{t}\right)}\right],
\end{aligned}
$$

where $\hat{P}_{X}$ is the momentum operator and $m$ the mass of particle $X$.

The main results here can be easily understood on physical grounds. Initially, the energy is independent of $y$ and simply the average of the energy of a Gaussian wave packet with zero group velocity, $E^{(0)}=\hbar^{2} / 8 m \sigma_{X}^{2}$ and that of a Gaussian wave packet with group velocity $k, E^{(k)}=E^{(0)}+\hbar^{2} k^{2} /(2 m)$. As time evolves and the wave packets begin to separate, the energy changes depending on the exact trajectory $Y_{t}$. These trajectories and the corresponding energies are plotted in Fig. 5. Once the wave packets are well separated, only one term in Eq. (44) is relevant and the wave function behaves as if effectively factorized (see Ref. [23] for a discussion on effective wave functions). As a consequence, the energy converges either to $E^{(0)}$ or $E^{(k)}$. The fact that the spreading energy $E^{(0)}$ remains constant is a consequence of the assumption that we can neglect the spreading of the wave packets for the duration of $\hat{U}_{Y}$. We also note that the average energy remains constant at all times as expected since there is no interaction term.

\section{CONCLUSIONS AND DISCUSSION}

In this paper we have determined the contributions to a quantum system's energy exchanges when it is coupled to an environment and externally driven. Going beyond the reduced system state picture, which cannot distinguish energy flows that arise from existing entanglement between system and environment or from their ongoing interactions, we have here used conditional wave functions that allow a single-shot analysis. Based on the CWF we have here derived a formally exact analytic expression for the energy exchanges of the system in a single run of an experiment, stated in Eq. (14), without restricting how the global Hamiltonian is dependent on time or the form of the interaction Hamiltonian.

The derivation reveals three distinctly different contributions: an external contribution, an interaction contribution, and an entanglement contribution, directly associated with entanglement between the system and the environment. Each of these contributions can be present on its own, e.g., when the system and environment are entangled but not interacting and $\hat{H}_{S}$ has no explicit time dependence, only the entanglement contribution is present. Naturally, in order to entangle the system with its environment there must have been an interaction. However, after such initial preparation, the interaction Hamiltonian can be switched off and the interaction contribution vanishes, but the entanglement contribution remains. This provides a direct link between entanglement and energy fluctuations in a single run of an experiment for the first time.

Taking the statistical average for these contributions, Eqs. (16) and (15), i.e., the average over many runs of the experiment, we have related the single-shot analysis to the expectation value of $\hat{H}_{S}(t)$, Eq. (19), and its time derivative, Eqs. (20) and (22). The external contribution yields the familiar expectation value of the Hamiltonian's explicit time dependence. The term containing the time dependence of the reduced density operator splits into the average of the interaction and entanglement contributions, Eq. (21), where the average of the entanglement contribution can only be nonzero if an interaction is present, in contrast to the singleshot case. This is in line with the expectation that there can be no average energy transfer due to entanglement alone. We have demonstrated the results with a number of concrete examples that help to provide an intuitive picture of energy flow in physical space. 
CWFs are closely related to weak values $[43,46,47]$ and can be reconstructed experimentally [37,38,40], making the conditional energy empirically accessible. An interesting open question is if the entanglement contribution could be experimentally used to quantify "quantumness," i.e., the appearance of entanglement between the system and the environment, and to what extent it may be linked to quantum advantages in thermodynamic processes.

Generalizing the presented results for the bare Hamiltonian to investigate the fluctuations associated with the interaction term in the Hamiltonian could open new methods to tackle strongly coupled quantum systems, and connect with known thermodynamic results. For example, the CWFs would allow one to identify effective energetic exchanges when one considers coarse-graining methods and effective Hamiltonians such as the Hamiltonian of mean force [52,62]. Given the success in addressing quantum transport in nanoelectronic systems, it would be interesting to relate the statistical description given here to thermodynamic notions of work and heat in these systems [63].

In the limit of weak interaction, continuous monitoring of the environment and markovian evolution, the entanglement contribution will be negligible. Thus, heat would be identified with the interaction contribution. For an entangled system and environment, an interesting prospect is to identify some form of "entanglement heat," that is, part of the energy exchanged with the environment that can be associated with entanglement.

Furthermore, the quantum to classical transition could also be studied by generalizing classical results $[57,58]$ and analyzing the limit of large quantum numbers, vanishing entanglement or center-of-mass dynamics [64].

\section{ACKNOWLEDGMENTS}

This work has been supported in part by the Academy of Finland through its Quantum Technology Finland CoE Project No. 312298. R.S. acknowledges the financial support by the Magnus Ehrnrooth Foundation as well as from the CMMP Education Network. J.A. acknowledges support from EPSRC (Grant No. EP/M009165/1) and the Royal Society. This research was supported by the COST network MP1209 "Thermodynamics in the quantum regime."

\section{APPENDIX: COARSE-GRAINING FOR MIXED STATES}

Following Ref. [61], Bohmian-like trajectories can equally be defined for unitary evolution of general mixed states $\hat{\sigma}(t)$. Indeed, for Hamiltonians of the form $\hat{H}=$
$\sum_{i=0}^{N} \sum_{j=1}^{3}\left[\hat{P}_{j}^{(i)^{2}} / 2 m^{(i)}\right]+V\left(\left\{\hat{Z}_{j}^{(i)}\right\}, t\right)$ the velocity field is formally equal to Eq. (1) in the main text, for $\hat{\sigma}(t)$ now a general mixed state rather than a pure state. The trajectories are then defined formally in the same way as in the main text. The natural generalization of the conditional energy is taken from Eq. (12) in the main text, namely

$$
u^{(\sigma)}\left(t \mid \vec{z}_{0}\right)=\frac{\operatorname{Tr}\left\{\hat{H}_{S}(t) \hat{\tilde{\rho}}^{(\sigma)}\left(t \mid \vec{z}_{0}\right)\right\}}{\operatorname{Tr}\left\{\hat{\tilde{\rho}}^{(\sigma)}\left(t \mid \vec{z}_{0}\right)\right\}}
$$

where $\hat{\tilde{\rho}}^{(\sigma)}\left(t \mid \vec{z}_{0}\right):=\left\langle\vec{Y}_{t}^{(\sigma)}|\hat{\sigma}(t)| \vec{Y}_{t}^{(\sigma)}\right\rangle_{E}$ defines an unnormalized conditional density matrix, the generalization of the conditional wave function [43], and $\vec{Y}_{t}^{(\sigma)}:=\vec{Y}^{(\sigma)}\left(\vec{z}_{0}, t\right)$ is a trajectory of the environment generated by $\hat{\sigma}(t)$ given the initial condition $\vec{z}_{0}$ using a notation analogous to the main text. The derivation now follows verbatim and we find the generalized quantities

$$
d_{t} u^{(\sigma)}\left(t \mid \vec{z}_{0}\right)=d_{t} u_{\mathrm{int}}^{(\sigma)}\left(t \mid \vec{z}_{0}\right)+d_{t} u_{\mathrm{ent}}^{(\sigma)}\left(t \mid \vec{z}_{0}\right)+d_{t} u_{\mathrm{ext}}^{(\sigma)}\left(t \mid \vec{z}_{0}\right),
$$

where

$$
\begin{gathered}
d_{t} u_{\text {int }}^{(\sigma)}\left(t \mid \vec{z}_{0}\right)=\frac{\operatorname{Tr}\left\{d_{t} \hat{\tilde{\rho}}_{\text {int }}^{(\sigma)}\left(t \mid \vec{z}_{0}\right)\left[\hat{H}_{S}(t)-u^{(\sigma)}\left(t \mid \vec{z}_{0}\right)\right]\right\}}{\operatorname{Tr}\left\{\hat{\tilde{\rho}}^{(\sigma)}\left(t \mid \vec{z}_{0}\right)\right\}} ; \\
d_{t} u_{\mathrm{cor}}^{(\sigma)}\left(t \mid \vec{z}_{0}\right)=\frac{\operatorname{Tr}\left\{d_{t} \hat{\tilde{\rho}}_{\text {cor }}^{(\sigma)}\left(t \mid \vec{z}_{0}\right)\left[\hat{H}_{S}(t)-u^{(\sigma)}\left(t \mid \vec{z}_{0}\right)\right]\right\}}{\operatorname{Tr}\left\{\hat{\tilde{\rho}}\left(t \mid Y_{t}\right)\right\}} ; \\
d_{t} u_{\mathrm{ext}}^{(\sigma)}\left(t \mid \vec{z}_{0}\right)=\frac{\operatorname{Tr}\left\{\hat{\tilde{\rho}}^{(\sigma)}\left(t \mid \vec{z}_{0}\right) d_{t} \hat{H}_{\mathrm{S}}(t)\right\}}{\operatorname{Tr}\left\{\hat{\tilde{\rho}}^{(\sigma)}\left(t \mid \vec{z}_{0}\right)\right\}},
\end{gathered}
$$

with $\quad d_{t} \hat{\tilde{\rho}}_{\text {int }}^{(\sigma)}=-\frac{l}{\hbar}\left\langle\vec{Y}_{t}^{(\sigma)}\left|\left[\hat{H}_{\text {int }}, \hat{\sigma}(t)\right]\right| \vec{Y}_{t}^{(\sigma)}\right\rangle_{E} \quad$ and $\quad d_{t} \hat{\tilde{\rho}}_{\text {cor }}^{(\sigma)}=$ $-\frac{l}{\hbar}\left(\left\langle\vec{Y}_{t}^{(\sigma)}\left|\left[\hat{H}_{E}, \hat{\sigma}(t)\right]\right| \vec{Y}_{t}^{(\sigma)}\right\rangle_{E}-v^{(y)} \cdot\left\langle\vec{Y}_{t}^{(\sigma)}\left|\left[\hat{\vec{P}}^{(y)}, \hat{\sigma}(t)\right]\right| \vec{Y}_{t}^{(\sigma)}\right\rangle_{E}\right)$. The only difference is that the entanglement term should now be understood as a correlations term since it will be nonzero for both classical and quantum correlations. Finally, the relations for the statistical averages also hold, namely

$$
\left\langle\left\langle u^{(\sigma)}(t)\right\rangle\right\rangle:=\int d \mathbf{z}\langle\vec{z}|\hat{\sigma}(0)| \vec{z}\rangle u^{(\sigma)}(t \mid \vec{z})=\operatorname{Tr}\left\{\hat{H}_{S}(t) \hat{\sigma}(t)\right\}
$$

and

$$
\begin{gathered}
\operatorname{Tr}\left\{\hat{\sigma}(t) d_{t} \hat{H}_{S}(t)\right\}=\left\langle\left\langle d_{t} u_{\mathrm{ext}}^{(\sigma)}(t)\right\rangle\right\rangle ; \\
\operatorname{Tr}\left\{\hat{H}_{S}(t) d_{t} \hat{\sigma}(t)\right\}=\left\langle\left\langle d_{t} u_{\mathrm{int}}^{(\sigma)}(t)\right\rangle\right\rangle+\left\langle\left\langle d_{t} u_{\mathrm{ent}}^{(\sigma)}(t)\right\rangle\right\rangle ; \\
\left\langle\left\langle d_{t} u_{\mathrm{ent}}^{(\sigma)}(t)\right\rangle\right\rangle=\int d \mathbf{z} u^{(\sigma)}(t, \vec{y})\left\langle\vec{x}\left|d_{t} \hat{\tilde{\rho}}_{\mathrm{int}}^{(\sigma)}\right| \vec{x}\right\rangle \\
=-\frac{l}{\hbar} \operatorname{Tr}\left\{\hat{\sigma}(t)\left[\hat{u}^{(\sigma)}(t), \hat{H}_{\mathrm{int}}\right]\right\} .
\end{gathered}
$$

[1] H.-P. Breuer and F. Petruccione, The Theory of Open Quantum Systems (Oxford University Press, Oxford, 2002).

[2] G. Lindblad, Non-Equilibrium Entropy and Irreversibility. (Springer, Amsterdam, 1983).
[3] A. Rivas and S. F. Huelga, Open Quantum Systems (Springer, Berlin, 2012).

[4] S. Vinjanampathy and J. Anders, Contemp. Phys. 57, 545 (2016). 
[5] F. W. J. Hekking and J. P. Pekola, Phys. Rev. Lett. 111, 093602 (2013).

[6] J. M. Horowitz, Phys. Rev. E 85, 031110 (2012).

[7] C. Elouard, D. A. Herrera-Martí, M. Clusel, and A. Auffèves, NPJ Q. Info. 3, 9 (2017).

[8] J. P. Pekola, P. Solinas, A. Shnirman, and D. V. Averin, New J. Phys. 15, 115006 (2013).

[9] J. P. Pekola, S. Suomela, and Y. M. Galperin, J. Low Temp. Phys. 184, 1015 (2016).

[10] R. Sampaio, S. Suomela, and T. Ala-Nissila, Phys. Rev. E 94, 062122 (2016).

[11] W. T. Strunz, L. Diósi, and N. Gisin, Phys. Rev. Lett. 82, 1801 (1999).

[12] N. Gisin and I. C. Percival, J. Phys. A 25, 5677 (1992).

[13] C. W. Gardiner, A. S. Parkins, and P. Zoller, Phys. Rev. A 46, 4363 (1992).

[14] K. Mølmer, Y. Castin, and J. Dalibard, J. Opt. Soc. Am. B 10, 524 (1993).

[15] G. Manzano, J. M. Horowitz, and J. M. R. Parrondo, Phys. Rev. X 8, 031037 (2018).

[16] M. Campisi, J. Pekola, and R. Fazio, New J. Phys. 17, 035012 (2015).

[17] Z. Gong, Y. Ashida, and M. Ueda, Phys. Rev. A 94, 012107 (2016).

[18] F. Liu and J. Xi, Phys. Rev. E 94, 062133 (2016).

[19] H. M. Wiseman and G. J. Milburn, Quantum Measurement and Control (Cambridge University Press, Cambridge, 2009).

[20] B. d'Espagnat, Conceptual Foundations of Quantum Mechanics (CRC Press, Boca Raton, FL, 2018).

[21] J. Gambetta and H. M. Wiseman, Phys. Rev. A 66, 012108 (2002).

[22] J. Gambetta and H. M. Wiseman, Phys. Rev. A 68, 062104 (2003).

[23] S. Teufel and D. Dürr, Bohmian Mechanics (Springer, Berlin, 2009).

[24] D. Bohm, Phys. Rev. 85, 166 (1952).

[25] D. Bohm, Phys. Rev. 85, 180 (1952).

[26] N. Makri, J. Phys. Chem. A 108, 806 (2004).

[27] W. Koch, F. Grossmann, and D. J. Tannor, Phys. Rev. Lett. 105, 230405 (2010).

[28] I. Burghardt and G. Parlant, J. Chem. Phys. 120, 3055 (2004).

[29] N. Makri and W. H. Miller, J. Chem. Phys. 116, 9207 (2002).

[30] E. R. Bittner, J. Chem. Phys. 119, 1358 (2003).

[31] E. Colomés, Z. Zhan, D. Marian, and X. Oriols, Phys. Rev. B 96, 075135 (2017).

[32] A. Abedi and M. J. Sharifi, J. Comput. Electron. 17, 68 (2018).

[33] X. Oriols, Phys. Rev. Lett. 98, 066803 (2007).

[34] Z. Zhan, E. Colomés, D. Pandey, S. Yuan, and X. Oriols, Phys. Rev. B 99, 155412 (2019).
[35] A. Sanz, X. Giménez, J. M. Bofill, and S. Miret-Artés, Chem. Phys. Lett. 478, 89 (2009).

[36] A. Sanz, D. López-Durán, and T. González-Lezana, Chem. Phys. 399, 151 (2012).

[37] D. H. Mahler, L. Rozema, K. Fisher, L. Vermeyden, K. J. Resch, H. M. Wiseman, and A. Steinberg, Sci. Adv. 2, e1501466 (2016).

[38] S. Kocsis, B. Braverman, S. Ravets, M. J. Stevens, R. P. Mirin, L. K. Shalm, and A. M. Steinberg, Science 332, 1170 (2011).

[39] C. Philippidis, C. Dewdney, and B. J. Hiley, Nuov. Cim. B (1979) 52, 15 (1979).

[40] Y. Xiao, Y. Kedem, J.-S. Xu, C.-F. Li, and G.-C. Guo, Opt. Express 25, 14463 (2017).

[41] B. Hiley and P. Van Reeth, Entropy 20, 353 (2018).

[42] A. Benseny, G. Albareda, Á. S. Sanz, J. Mompart, and X. Oriols, Eurphys. J. D 68, 286 (2014).

[43] T. Norsen and W. Struyve, Ann. Phys. 350, 178 (2014).

[44] P. R. Holland, The Quantum Theory of Motion: An Account of the de Broglie-Bohm Causal Interpretation of Quantum Mechanics (Cambridge University Press, Cambridge, 1995).

[45] A. B. Nassar and S. Miret-Artés, Bohmian Mechanics, Open Quantum Systems and Continuous Measurements (Springer, Berlin, 2017).

[46] R. Flack and B. Hiley, Entropy 20, 367 (2018).

[47] Y. Aharonov, D. Z. Albert, and L. Vaidman, Phys. Rev. Lett. 60, 1351 (1988).

[48] R. Sampaio, S. Suomela, T. Ala-Nissila, J. Anders, and T. G. Philbin, Phys. Rev. A 97, 012131 (2018).

[49] J. Anders and H. J. D. Miller, New J. Phys. 19, 062001 (2017).

[50] P. Solinas, D. V. Averin, and J. P. Pekola, Phys. Rev. B 87, 060508(R) (2013).

[51] P. Talkner and P. Hänggi, Phys. Rev. E 93, 022131 (2016).

[52] M. Campisi, P. Talkner, and P. Hänggi, Phys. Rev. Lett. 102, 210401 (2009)

[53] J. J. Alonso, E. Lutz, and A. Romito, Phys. Rev. Lett. 116, 080403 (2016).

[54] T. G. Philbin and J. Anders, J. Phys. A 49, 215303 (2016).

[55] U. Seifert, Phys. Rev. Lett. 116, 020601 (2016).

[56] C. Jarzynski, Phys. Rev. X 7, 011008 (2017).

[57] P. Strasberg and M. Esposito, Phys. Rev. E 95, 062101 (2017).

[58] H. J. D. Miller and J. Anders, Phys. Rev. E 95, 062123 (2017).

[59] E. Aurell, Phys. Rev. E 97, 042112 (2018).

[60] H. J. D. Miller and J. Anders, Nat. Commun. 9, 2203 (2018).

[61] D. Dürr, S. Goldstein, R. Tumulka, and N. Zanghí, Found. Phys. 35, 449 (2005).

[62] J. G. Kirkwood, J. Chem. Phys. 3, 300 (1935).

[63] M. F. Ludovico, M. Moskalets, D. Sánchez, and L. Arrachea, Phys. Rev. B 94, 035436 (2016).

[64] X. Oriols and A. Benseny, New J. Phys. 19, 063031 (2017). 